\title{
THE SEPARATION OF ARSENIC FROM ANTIMONY AND OTHER METALS, WITH SOME APPLICATIONS TO TOXICOLOGICAL WORK.*
}

\author{
By STANLEY W. COLLINS, B.Sc., F.I.C.
}

(Read at the Meeting, April 3, 1912.)

Friedheim and Michaelis (Ber., 1895, 28, 1414) have described a method for quantitatively separating arsenic from vanadium, molybdenum, and tungsten, by distillation with methyl alcohol in a current of hydrogen chloride. Under these conditions it is stated that arsenic trioxide forms a volatile methyl ester. In order to estimate the arsenic the distillate was treated with nitric acid and evaporated to dryness. The residue was dissolved in water, the solution made alkaline with ammonia, and the

* This work was carried out under the provisions of the Analytical Investigation Scheme. 
arsenic precipitated by magnesia mixture and weighed as magnesium pyroarsenate . In estimating arsenic pentoxide no reducing agent need be added, as it is reduced in । presence of methyl alcohol and hydrogen chloride, with the formation of the volatile , methyl arsenious ester.

Cantoni and Chautems (Annal. de Chim. anal., 1905, 10, 213-214) proposed to separate arsenic from antimony by passing a current of dry air at the ordinary temperature through a solution of arsenic trioxide in hydrochloric acid to which methyl alcohol had been added. They state that under these conditions the arsenic can be completely distilled, leaving the antimony in the residue. It appeared desirable to determine the possible application of these methods to the separation of arsenic from antimony, particularly when these elements are present with organic matter.

The following form of apparatus (see illustration, p. 231) was devised and found to be satisfactory for the experiments involving distillations :

A pear-shaped distilling flask of about 300 c.c. capacity, with a wide neck about $4 \mathrm{cms}$. diameter and $8 \mathrm{cms}$. long, drawn down at the top for a small cork or ground joint through which passes the tube for delivery of hydrogen chloride. The flask is provided with a wide side-tube, $A$, about $1 \mathrm{~cm}$. internal diameter, at about $4 \mathrm{cms}$. from the lower end of the neck. This tube is bent upwards at an angle of about 60 degrees with the neck, so that during distillation it is inclined upwards from the flask, which makes an angle of about 30 degrees with the vertical. This arrangement allows of rapid distillation, whilst it prevents the solution being carried over by spurting. The inlet tube $B$, of about $1 \mathrm{~cm}$. diameter and $2 \mathrm{cms}$. length at the lower end of the neck, serves for the introduction of substances to be analysed, and into it, before the distillation is commenced, a tap-funnel is fitted by means of a cork or a ground joint, to allow of the addition of more methyl alcohol. In a later form of apparatus the inlet tube $B$ was enlarged to about $1 \frac{1}{2} \mathrm{cms}$. diameter, thus making it more convenient for the introduction of substances to be analysed. The tap funnel was also ground in. The side-tube was ground into a Liebig's condenser, and bent so that, during an experiment, the condenser is in a position slightly sloping away from the distilling flask. The inside tube of the condenser is about $1 \mathrm{~cm}$. in diameter and $40 \mathrm{cms}$. in length, passing through a cork into a 30-ounce conical flask. Through another hole in the cork a tube filled with glass beads is fitted. The jacket of the condenser is about $17 \mathrm{cms}$. long.

Several experiments were carried out on the lines proposed by Cantoni and Chautems (loc. cit.), but the method proved to be of no value; for under the conditions described by these chemists only a small proportion of arsenic trioxide could be distilled, even when a current of dry air was passed for four hours. A more successful experiment was carried out by the method of Friedheim and Michaelis (loc. cit.). 0.2 grm. of arsenic trioxide was washed into the distillation flask with 50 c.c. of methyl alcohol, which was then saturated with dry hydrogen chloride. The flask was then heated on a water-bath; the distillation was repeated twice with 50 c.c. of methyl alcohol, a slow stream of hydrogen chloride being passed during the distillation. On diluting the residue in the flask and passing in sulphuretted hydrogen, no arsenious sulphide was precipitated. The method used in this and many subsequent experi- 
METALS, WITH SOME APPLICATIONS TO TOXICOLOGICAL WORK 231

ments for the estimation of arsenic in the distillate, consisted of saturating with sulphuretted hydrogen, after diluting with an equal volume of water, washing the precipitated arsenious sulphide with hot water, alcohol, a mixture of carbon disulphide and alcohol, alcohol, and then hot water. The precipitate was then washed from the

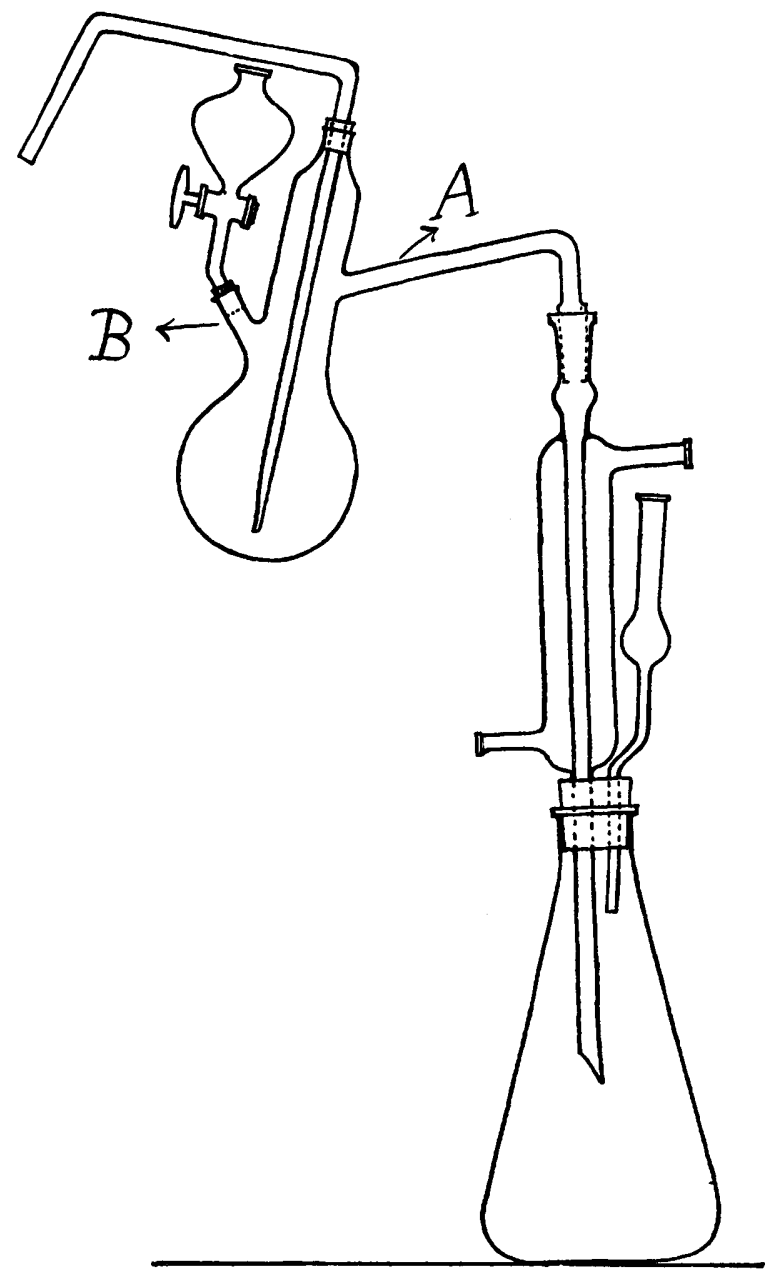

filter-paper finto a weighed dish, dried over a water-bath and then at $105^{\circ}$ to $110^{\circ} \mathrm{C}$., and weighed.

The precipitate was tested for antimony by digesting with strong hydrochloric acid, filtering, diluting and saturating with sulphuretted hydrogen. The antimony sulphide was dissolved in hydrochloric acid with a little potassium chlorate, and antimony obtained from the solution by means of pure iron wire.

Arsenic in the distillate was also estimated satisfactorily by titration with standard iodine solution. An experiment was made on $0.2 \mathrm{grm}$. of arsenic trioxide 
with 0.2 grm. of antimony trioxide, with the result that about 0.02 grm. of antimony trioxide was found with the distilled arsenious oxide. It will thus be seen that the formation of antimony chloride and its subsequent volatility render the method inapplicable to the quantitative separation of arsenic from antimony. Many experiments were made in order to overcome this difficulty, in the course of which it was found that the volatility of arsenic trioxide in the vapour of methyl alcohol was considerably increased by the addition of strong sulphuric acid or phosphorus pentoxide to the arsenic trioxide and methyl alcohol before distillation; neither arsenic pentoxide nor antimony trioxide could be distilled under these conditions. Attempts were made to devise a method based on these facts for the quantitative separation of arsenic from antimony, but it was found that the removal of the last traces of arsenic was a tedious process, in which it was necessary to use a comparatively large volume of methyl alcohol. A complete separation was, however, effected by taking a mixture of methyl alcohol and the oxides and a sufficient quantity of sulphuric acid, and passing a stream of hydrogen chloride through for some time before commencing the heating. The passage of the gas was continued throughout the distillation. Under these conditions it was found that the arsenic was completely distilled, whilst the more basic antimony was retained by the sulphuric acid. When phosphorus pentoxide was substituted for sulphuric acid in these experiments, small quantities of antimony were carried over with the arsenic:

The following account of typical experiments illustrates the application of this method:

0.08 grm. of arsenic trioxide and 0.08 grm. of antimony trioxide were washed into the distilling flask with 30 c.c. of methyl alcohol and a mixture of 6 c.c. of sulphuric acid (sp. gr. 1.84) with 20 c.c. of methyl alcohol. Hydrogen chloride was passed in for ten minutes and the flask heated on a water-bath, the distillate being collected in a 30-ounce conical flask into which a small quantity of water was occasionally poured over the glass beads. The distillation was repeated twice with 50 c.c. of methyl alcohol. The arsenic in the distillate was precipitated by sulphuretted hydrogen, washed, and weighed; 0.0796 grm. was found. No antimony sulphide could be detected in the precipitate. This analysis was repeated, using $0.2 \mathrm{grm}$. of arsenious oxide and $0.2 \mathrm{grm}$. of antimony oxide with 8 c.c. of sulphuric acid (sp. gr. 184). 0.200 grm. of arsenic trioxide was found. In the next experiment the conditions were the same, with the exception that the arsenic in the distillate was titrated with iodine, after adding an excess of sodium bicarbonate. $0.200 \mathrm{grm}$. of arsenic trioxide was found.

2.0 grms. of antimony oxide with 0.1 grm. of arsenic trioxide were mixed with 15 c.c. of sulphuric acid (sp. gr. 1.84) and 50 c.c. of methyl alcohol. The distillation was repeated with the following volumes of methyl alcohol, 50 c.c., 50 c.c., 20 c.c., 20 c.c., when, on testing the distillate with sulphuretted hydrogen, no precipitate was obtained. $0.1002 \mathrm{grm}$. of arsenic trioxide was found. The precipitate was free from antimony.

3.0 grms. of antimony oxide with 0.1 grn. of arsenic trioxide were mixed with 15 c.c. of sulphuric acid (sp. gr. 1.84) and 50 c.c. methyl alcohol. Distillation was repeated as in the last experiment. $0.101 \mathrm{grm}$. of arsenic trioxide was found. In this 
case the precipitate contained antimony. The experiment was repeated, using 3.0 grms. of antimony oxide with 0.06 grm. of arsenic trioxide and a smaller quantity of sulphuric acid-i.e., 10 c.c. A precipitate equivalent to 0.064 grm. of arsenic trioxide was found. Antimony was present in the precipitate.

The application of this method to the separation of arsenic pentoxide from antimony was next investigated. It was found that the arsenic from arsenic pentoxide could be distilled completely only in the presence of a reducing agent such as ferrous chloride. The following experiments show the results obtained for the estimation of arsenic pentoxide; the first one gave a satisfactory result for the separation of arsenic from other metals of the sulphuretted hydrogen group as well as from antimony:

$0.06 \mathrm{grm}$. of arsenic pentoxide with $0.05 \mathrm{grm}$. of antimony oxide, $0.04 \mathrm{grm}$. of bismuth oxide, $0.03 \mathrm{grm}$. of mercurous chloride, $0.03 \mathrm{grm}$. of mercuric chloride, and 5 grms. of ferrous chloride, were introduced into the distilling flask with 50 c.c. of methyl alcohol. To these were added 10 c.c. of sulphuric acid (sp. gr. 1.84); hydrogen chloride was passed through the contents of the flask before and during the distillation, which was repeated twice with 50 c.c. and twice with 20 c.c. of methyl alcohol. The distillate was saturated with sulphuretted hydrogen and the precipitated arsenious sulphide, washed, dried, and weighed, as before. The weight of arsenic pentoxide found was $0.0596 \mathrm{grm}$. The precipitated arsenious sulphide was found to be free from antimony, bismuth, and mercury.

$0.1 \mathrm{grm}$. of arsenic pentoxide with 2 grms. of antimony oxide was mixed in the distillation flask with 15 c.c. of sulphuric acid (sp. gr. 1.84), 50 c.c. of methyl alcohol and 5 grms. of ferrous chloride. Hydrogen chloride was passed before and during distillation, which was repeated twice with 50 c.c. and twice with 20 c.c. of methyl alcohol. The weight of arsenic pentoxide found was $0.0998 \mathrm{grm}$., and there was no antimony in the precipitate.

These results indicate that this method is applicable, under suitable conditions, to the quantitative separation of arsenic from antimony and from other metals. Before pointing out the conditions necessary for this method, it is essential to give an account of an experiment which shows the influence of water on the volatilisation of antimony trichloride: $1 \mathrm{grm}$. of antimony oxide was mixed with 10 c.c. of sulphuric acid (sp. gr. 1.84) and 50 c.c. of water. Hydrogen chloride was passed in for about fifteen minutes as well as during the distillation. Sulphuretted hydrogen was passed into the distillate, which yielded an appreciable precipitate of antimony sulphide. To about 20 c.c. of the residual solution an equal volume of methyl alcohol was added, and hydrogen chloride passed through as before. Separate small volumes of the distillate were collected and saturated with sulphuretted hydrogen. A barely perceptible coloration was obtained in the first portions, but, as the alcohol was distilled off, the quantity of antimony sulphide precipitated became appreciable. It is evident, therefore, that the material used in this method should be sufficiently free from water, since, in presence of water, sulphuric acid fails to retain the antimony completely. With regard to the amount of sulphuric acid necessary to retain the antimony, 10 c.c. of acid (sp. gr. 1.84) is a convenient quantity for not more than 1 grm. of antimony oxide; whilst 15 c.c. of acid 


\section{COLLINS: SEPARATION OF ARSENIC FROM ANTIMONY AND OTHER}

will retain up to 2 grms. of antimony oxide, and is the maximum quantity convenient to use with 50 c.c. of methyl alcohol, since with more than this amount the distillation becomes too slow. An experiment was next made in order to gain some idea of the maximum amount of arsenic trioxide which could be volatilised, using 50 c.c. of methyl alcohol. Arsenic trioxide 5 grms., with 10 c.c. of sulphuric acid, and 50 c.c. of methyl alcohol, were distilled in a stream of hydrogen chloride in the usual manner, and $0.96 \mathrm{grm}$. of arsenic trioxide was found to have been volatilised, an amount largely in excess of that used in any estimation. In the quantitative separation of arsenic from antimony, when not more than 0.2 grm. of arsenic trioxide was used, about 60 per cent. of the arsenic was volatilised in the first 50 c.c. of alcohol; a further 50 c.c. generally carried over all but a small amount, which was volatilised in the last 50 c.c. In presence of a large excess of antimony, it is advisable to add a further 10 c.c. of methyl alcohol and to test the distillate with sulphuretted hydrogen, as an additional 10 or 20 c.c. of methyl alcohol may be necessary to remove the last traces of arsenic.

Pure methyl alcohol, not specially dehydrated, was used in all these experiments. Commercial methyl alcohol is unsatisfactory, as the acids employed induce the formation of compounds of high boiling-point which retard the volatilisation of the arsenic. In an experiment in which ethyl alcohol was used, instead of methyl alcohol, the quantity of arsenic carried over was about half as great as would have been volatilised by the same volume of methyl alcohol.

It has been stated in this paper that the quantity of arsenic trioxide which could be distilled over was increased by mixing sulphuric acid with the alcohol and the oxide in the distillation flask, and that neither arsenic pentoxide nor antimony trioxide could be distilled in this manner. This suggests an easy test for arsenic trioxide in the presence of antimony as well as other metals. This method could only be applied as a test for arsenic trioxide in the presence of arsenic pentoxide when certain organic and other reducing agents were excluded.

The following experiments were made to test this method. Equal weights of the following substances were mixed-mercurous chloride, mercuric oxide, stannous chloride, cadmium oxide, antimony oxide. About $1 \mathrm{grm}$. of this mixture was placed in a small distilling flask, and 20 c.c. of methyl alcohol and 3 c.c. of sulphuric acid were added. The flask was attached to a condenser and heated by a flame, the distillate being collected in a small flask containing about 5 c.c. of water. On passing sulphuretted hydrogen through the distillate no precipitate was obtained. This experiment was repeated, using $1 \mathrm{grm}$. of the mixed substances with $0.3 \mathrm{grm}$. of arsenic pentoxide. No precipitate was obtained on passing sulphuretted hydrogen through the distillate. The experiment was again repeated, using $1 \mathrm{grm}$. of the mixed substances with 2 mgrms, of arsenic trioxide. A precipitate of arsenious sulphide was obtained on passing sulphuretted hydrogen through the distillate. 


\section{Application of this Method to the Separation of Arsenic from Antimony and Other Metals in the Presence of Organic Matter.}

The character of the material for analysis will determine the mode of application. The separation can be effected without previous destruction of the organic matter if the arsenic be not intimately combined with the organic matter. The material should also be fairly dry.

The following experiment is an example of these conditions : 20 grms. of powdered biscuit material were placed in the distillation flask; $0.1 \mathrm{grm}$. of arsenic trioxide and $0.1 \mathrm{grm}$. of antimony oxide were washed into the flask with a mixture of 50 c.c. of methyl alcohol and 8 c.c. of sulphuric acid. Hydrogen chloride was then passed through the contents of the flask for about ten minutes. The flask was beated on the water-bath, a stream of hydrogen chloride being continued during the distillation, which was repeated twice with 50 c.c. and twice with 20 c.c. of methyl alcohol. The arsenic was precipitated from the distillate by means of sulphuretted hydrogen, washed, dried, and weighed, and an amount equivalent to $0.0986 \mathrm{grm}$. of arsenic trioxide was found.

For the next experiments I obtained specimens of viscera from a case which had been treated with salvarsan. In the first test 50 grms. of the kidney were heated in a Jena distillation flask with sulphuric acid, and the water distilled off as completely as possible. Then methyl alcohol was added and hydrogen chloride passed through the viscid contents of the flask, and the distillation effected as before. This method was not satisfactory. Ninety-eight grms. of the liver were treated with 10 c.c. of nitric acid (sp. gr. 1.4), and charred in the usual manner with sulphuric acid. The charred mass was washed into the distilling flask with 50 c.c. of methyl alcohol, and about 5 grms. of ferrous chloride were added. Hydrogen chloride was then passed in as usual, and the flask heated on the water-bath. The distillation was repeated twice with 50 c.c. and twice with 20 c.c. of methyl alcohol. The distillate was saturated with sulphuretted hydrogen, and the precipitated arsenious sulphide was purified by dissolving in ammonia and reprecipitating. The arsenious sulphide was then dissolved in nitric acid, the solution made alkaline with ammonia, and the arsenic precipitated as magnesium ammonium arsenate, and finally weighed as the pyroarsenate. Weight of arsenic trioxide per 100 grms. of the liver : From the sulphide, 3.6 mgrms. ; from the pyroarsenate, $3 \cdot 1$ mgrms. The residue was found to be free from arsenic.

I am indebted to Mr. John Webster for the result of an estimation of arsenic in another portion of this liver. This estimation was carried out, in Dr. Willcox's laboratory, by the usual extraction method, the arsenic being finally weighed as magnesium pyroarsenate. Weight of arsenic trioxide found per 100 grms. of viscera was 3.3 mgrms. This result was confirmed by an estimation made by the MarshBerzelius method.

Attempts to confirm the statement of Friedheim and Michaelis (loc. cit.), that the volatility of arsenic trioxide in methyl alcohol and hydrogen chloride is due to the formation of a methyl arsenious ester, were unsuccessful. An excess of arsenic trioxide was heated with dehydrated methyl alcohol and sulphuric acid for some hours, also methyl alcohol with arsenic trioxide was treated with hydrogen chloride, and also 
with hydrogen chloride and sulphuric acid. In each experiment the distillate was fractionally redistilled, with the result that the boiling-point did not rise appreciably above that of methyl alcohol. The compounds of arsenic with alcohol radicles have been investigated by the following: J. M. Crafts (Bull. Soc. Chim., 1870, 14, 99) obtained arsenates and arsenites of the alkyl radicles of the general formulæ $\mathrm{R}_{3} \mathrm{AsO}_{4}$ and $\mathrm{R}_{3} \mathrm{AsO}_{3}$. For the arsenites he used three methods involving the interaction in a sealed tube of (1) ethyl iodide and silver arsenite at $150^{\circ} \mathrm{C}$; (2) arsenic bromide and sodium ethoxide; (3) arsenic trioxide and ethyl silicate at $200^{\circ} \mathrm{C}$. The boiling-point of methyl arsenite is $128^{\circ} \mathrm{C}$. Auger (Compt. rend., 1902, 134, 238, and $1906,143,907)$ states that he obtained small yields of esters by heating arsenic trioxide and alcohol together in a sealed tube for some hours, although he did not succeed in isolating the esters. The maximum amount of esterification in the case of methyl alcohol is given as 6.5 per cent. The method of estimation of the amount of esterification appears not to be very satisfactory (cf. J. Chem. Soc., 1908, 93, 1367). In experimenting with propyl, normal butyl, and isobutyl alcohol with arsenic trioxide, he states that good results were obtained by removing the water formed either by fractional distillation or by passing the alcohol and water vapour over calcium carbide in an adapter, when the water is removed and the alcohol returns to the flask in which the reaction is taking place.

W. R. Lang, J. T. Mackey, and R. A. Gortner (J. Chem. Soc., 1908, 93, 1364) obtained a yield of 33.8 per cent. of methyl arsenite by heating methyl alcohol and arsenic trioxide together in the presence of anhydrous copper sulphate under a reflex condenser, and fractionating the resulting liquid under diminished pressure. They state that they have prepared methyl arsenite by a similar method without the anhydrous copper sulphate. No statement is made as to yield obtained.

J. T. Mackey (J. Chem. Soc., 1909, 95, 605) prepared esters of the aliphatic alcohols with antimony trioxide, using anhydrous copper sulphate as a dehydrating agent. The boiling-point of methyl antimonite is given as $65^{\circ} \mathrm{C}$.

Some esterification appears therefore to take place when arsenic trioxide and methyl alcohol are heated together, the amount of ester formed being increased in the presence of a desiccating substance such as anhydrous copper sulphate. However, under the conditions which prevailed during these estimations no evidence has been obtained for the formation of an arsenious ester. I propose to further examine the possibility of ester formation involved in the separation described in this communication.

In conclusion, I desire to express my thanks to Dr. W. H. Willcox and Mr. John Webster for their practical interest and help, and to Mr. A. Chaston Chapman for the help he has afforded me throughout the course of this work.

Chemical Department, King's College, London. 


\section{Discussion.}

The President, having invited discussion,

Mr. Chaston Chapman suggested that the function of the sulphuric acid might be not only to retain the more basic antimony, but also to assist esterification. He did not quite know upon what evidence Mr. Collins based his opinion that no formation of ester occurred, and he (Mr. Chapman) could not help feeling that the separation was in reality dependent on the formation of a methyl arsenious ester. With the comparatively small quantities of arsenic used in Mr. Collins' experiments, the detection in the distillate of such an ester would not be easy; but it was quite clear that its formation would be favourably influenced by the presence of the sulphuric acid.

Mr. C. Revis said that this process seemed to be a great advance on that of Clark. He had had occasion to use Clark's process a good deal some years ago, but had not been able to obtain very satisfactory results with it, finding that antimony was carried over during the distillation, especially when the antimony was large in quantity as compared with the arsenic. When, however, no other metal but arsenic was present, the results were fairly good.

Dr. Willcox said the process was itself an old one, and he (Dr. Willcox) believed that Taylor had claimed to be the originator of a modification of it for toxicological purposes. The process in that form, however, involved considerable risk of explosion, and it was not reliable for the separation of arsenic from small quantities of antimony and tin. Mr. Collins had tested his form of the process very thoroughly, and he (Dr. Willcox) believed that, if carried out under the conditions which Mr. Collins laid down, it would be found to be reliable for the separation of arsenic from antimony, mercury, bismuth, etc. It would be very useful for toxicological work, especially in criminal cases of arsenical poisoning, because in such cases doses of bismuth were frequently given, and there was considerable difficulty in the analysis of viscera containing arsenic accompanied by large quantities of bismuth when the usual method of precipitation as sulphides was employed. Apart from the question of the separation of arsenic from antimony, etc., this process would be valuable in dealing with arsenic alone, as was shown by the results that had been obtained in the case of salvarsan-poisoning referred to.

Mr. T. Macara said that the process devised by the late Dr. Clark was intended for use in connection with white metals, bronzes, and similar materials, and for such purposes it gave very good results when properly applied. It was essential to use strong hydrochloric acid, and not to concentrate the solution too much and so unduly raise its boiling-point. The one fault of the process was that it necessitated two or three distillations, but that difficulty might be overcome by passing hydrochloric acid gas into the distilling flask, as suggested by $\mathrm{Mr}$. Collins, which would enable good results to be obtained at a comparatively low temperature.

The President said that it seemed to him that Mr. Collins' process was essentially different from that of Dr. Clark. Of course, in a toxicological investigation it would not be proper to assume that a volatile product was arsenic without weighing it as a definite arsenical compound; but for many purposes it would probably be 
sufficient to boil the sulphide of arsenic with water, and titrate the arsenious oxide with iodine.

Mr. CoLLins, in reply, said that the statement of Frankheim and Michaelis was that the method depended on the formation of a methyl arsenious ester, but so far as his own work had gone he had not been able to obtain any evidence of the formation of such an ester. He had, however, only as yet been able to make one experiment as to this, which showed that the boiling-point of the distillate was not higher than that of methyl alcohol, whereas the boiling-point of pure methyl arsenite was $128^{\circ} \mathrm{C}$. In this experiment dehydrated methyl alcohol and sulphuric acid were heated with arsenic trioxide, and hydrochloric acid gas was passed through; on fractional distillation it was found that the boiling-point did not rise appreciably above that of methyl alcohol. He hoped later on to make further experiments by carrying out the fractional distillation under reduced pressure, under which conditions some esterification might possibly take place.

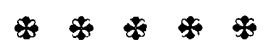

